\title{
Healthcare costs and resource utilization of asthma in Germany: a claims data analysis
}

\author{
Christian Jacob $\cdot$ Benno Bechtel $\cdot$ Susanne Engel $\cdot$ \\ Peter Kardos • Roland Linder · Sebastian Braun • \\ Wolfgang Greiner
}

Received: 28 July 2014/ Accepted: 6 February 2015 / Published online: 26 February 2015

(C) Springer-Verlag Berlin Heidelberg 2015

\begin{abstract}
Introduction Asthma is associated with a substantial economic burden on the German Statutory Health Insurance. Aims and objectives To determine costs and resource utilization associated with asthma and to analyze the impact of disease severity on subgroups based on age and gender. Methods A claims database analysis from the statutory health insurance perspective was conducted. Patients with an ICD-10-GM code of asthma were extracted from a $10 \%$ sample of a large German sickness fund. Five controls for each asthma patient matched by age and gender were randomly selected from the same database. Costs and resource utilization were calculated for each individual in the asthma and control group. Incremental asthma-related costs were calculated as the mean cost difference. Based on prescribed asthma medication, patients were classified as intermittent or persistent. In addition, age groups of $\leq 5$,
\end{abstract}

C. Jacob $(\bowtie) \cdot$ S. Braun

Xcenda GmbH, Lange Laube 31, 30159 Hannover, Germany

e-mail: christian.jacob@xcenda.de

B. Bechtel

GlaxoSmithKline, Uxbridge, Greater London, UK

S. Engel $\cdot$ R. Linder

Scientific Institute of TK for Benefit and Efficiency in Health

Care, Hamburg, Germany

\section{P. Kardos}

Group Practice and Centre for Pneumology Allergy and Sleep Medicine at Red Cross Maingau Hospital, Frankfurt Am Main, Germany

W. Greiner

Faculty of Health Sciences, University of Bielefeld,

Bielefeld, Germany
$6-18$, and $>18$ years were analyzed separately and gender differences were investigated.

Results Overall, 49,668 individuals were included in the asthma group. On average, total annual costs per patient were $€ 753$ higher $(p=0.000)$ compared to the control group ( $€ 2,168$ vs. $€ 1,415)$. Asthma patients had significantly higher $(p=0.000)$ outpatient $(€ 217)$, inpatient $(€ 176)$, and pharmacy costs (€259). Incremental asthmarelated total costs were higher for patients with persistent asthma compared to patients with intermittent asthma $(€ 1,091$ vs. $€ 408)$. Women aged $>18$ years with persistent asthma had the highest difference in costs compared to their controls $(€ 1,207 ; p<0.0001)$. Corresponding healthcare resource utilization was significantly higher in the asthma group $(p=0.000)$.

Conclusions The treatment of asthma is associated with an increased level of healthcare resource utilization and significantly higher healthcare costs. Asthma imposes a substantial economic burden on sickness funds.

Keywords Asthma - Claims data - Cost of illness . Disease severity $\cdot$ Persistent $\cdot$ Intermittent

JEL Classification I10

\section{Introduction}

Asthma is a chronic inflammatory disorder of the airways and one of the most common chronic diseases in Germany. About $10 \%$ of the pediatric population is suffering from asthma [1-4] followed by adults with about $5 \%$ [5-8]. The prevalence of asthma in the Statutory Health Insurance (SHI) is approximately $6 \%$ [2]. Depending on the severity of the disease, asthma poses a considerable burden on 
affected individuals, resulting in loss of productivity and participation in family life [9]. The disease also represents a constant major economic burden for the German statutory healthcare system $[2,10,11]$ with approximately $€ 1.789$ billion in 2008, accounting for $0.7 \%$ of the total healthcare expenditure in Germany [12]. Several studies have assessed the costs of asthma in the German setting, considering a payer's perspective or a societal perspective $[2,5,10,11$, 13-16]. Yet, further research is required as data of the available studies is fragmentary and does not include all relevant cost domains. Due to methodological limitations, the reported results for the resource use and costs strongly rely on assumptions, which are associated with uncertainty. Moreover, most studies used cost data that can be considered as obsolete reaching from 1992 to 2000 as reference years for cost calculation. Former studies reported disease severity as a considerable factor, with a significant impact on total asthma costs $[11,17]$. Hence, a detailed analysis of the influence of disease severity on resource use and costs is advisable. The aim of this study was to identify resource utilization for patients with asthma and the average disease related costs on an individual patient level. Further objectives were to analyze the impact of disease severity for subgroups based on age and gender, which is still lacking for the German setting [18].

\section{Materials and methods}

Data and study population

Claims data from German sickness funds include age and gender of the insured individual and detailed reimbursementrelated data for outpatient care, inpatient care, pharmaceuticals, therapeutic devices, rehabilitation, and sick leave. Every single healthcare service reimbursed by the sickness fund can be identified and analyzed. All information on the different healthcare services can be linked on an individual patient level via a unique identification code. [19].

Anonymized claims data from the largest German sickness fund (Techniker Krankenkasse) were analyzed. The sickness fund covered approximately 8 million persons in 2010. Patients with a diagnosis code of asthma (International Statistical Classification of Diseases and Related Health Problems, 10. Revision, German Modification ICD10-GM; Asthma bronchiale: J45.0, J45.1, J45.8, J45.9; Status asthmaticus: J46) were identified in the inpatient or outpatient setting. Primary and secondary diagnosis codes in the inpatient setting and confirmed diagnosis codes in the outpatient setting were taken into account. The criterion "confirmed" is an additional attribute in the outpatient data. It clarifies the certainty of the diagnosis. The study period was from January 1, 2010 to December 31, 2010.
Patients were required to be continuously insured within the study period. Individuals who died in the study period were excluded from the analysis. Due to data protection regulations, a $10 \%$ random sample of all identified asthma patients was used for further analyses.

To reflect disease severity, all asthma patients were stratified as having either intermittent or persistent asthma. The classification was based on prescribed asthma medication. Patients with a record of any of certain asthma medications (long-acting $\beta_{2}$-agonist (LABA; ATC: R03AC13, R03AK07, R03AK72, R03AC12, R03AK61, R03AK06, R03CC12, R03CC13, R03CC14, R03CC63, R03AK71), leukotriene modifiers (LTRA, ATC: R03DC03), inhaled corticosteroid (ICS; ATC: R01AD01, R03BA01, R03BA02, R03BA08, R03BA05, R03BA07), oral corticosteroid (OCS; ATC: H02AB03, H02AB04, H02AB07, H02AB06, H02AB56, H02AB08), Anti-IgE; ATC: R03DX05), Theophylline (ATC: R03DA04, R03DA54), and ipratropium bromide (ATC: R03BB01) or a documented hospitalization with a primary diagnosis of asthma (ICD-10-GM; Asthma bronchiale: J45.0, J45.1, J45.8, J45.9; Status asthmaticus: J46) were classified as having persistent asthma; whereas patients receiving only reliever medication (i.e. at least one prescription of a shortacting 32 -agonist; ATC: R03AC04, R03AK03, R03AK05, R03AC02, R03CC02, R03AC03, R03CC03) or no asthma specific medication were classified as having intermittent asthma.

In addition to the observation of the whole study population, different age groups of $\leq 5,6-18$, and $>18$ years were analyzed. Moreover, differences in gender groups were investigated.

\section{Control group design}

A randomly selected control group of individuals without asthma was extracted from the population of the participating sickness fund. To ensure the control group had no history of asthma, these insured persons were required to have an asthma diagnosis-free record for the time of the study period and the 2 years prior to that time frame. Five controls were matched exactly by year of birth and gender to each selected asthma patient.

\section{Calculation of costs and healthcare resource utilization}

Costs and healthcare resource utilization (HRU) were calculated from the perspective of the statutory health insurance. Patient co-payments or out-of-pocket payments were not considered. German healthcare insurance covers almost all accruing costs [20]. All costs were calculated on an annual scale for each individual patient in the asthma and in the control group. Costs were calculated separately for each of the six domains-outpatient care, inpatient care, 
pharmaceuticals, therapeutic devices and remedies, rehabilitation, and sick leave payments to identify potential cost drivers. Outpatient care covers all costs for services performed in an outpatient setting. Inpatient care summarizes all costs of performed services and administered drugs during inpatient stays. Pharmaceuticals include the costs of drug prescriptions in the outpatient setting. Therapeutic devices (Hilfsmittel) are devices such as walkers and wheelchairs to support the patient in recovering and everyday care. Remedies (Heilmittel) are services such as massages or occupational therapy provided by medically trained personal. The costs of rehabilitation are covered by the sickness fund for individuals who are not part of the workforce such as children and retirees. The costs of sick leave payments are covered by the sickness funds for employees beginning with the seventh week of sick leave, the first 6 weeks have to be paid by the employer. Total costs were calculated as the sum of the six domains. Asthma-related costs were calculated as the mean cost difference between the costs of the asthma group and the matched control group (incremental approach).

The reimbursement of services in the outpatient care setting in Germany is regulated by the Uniform Valuation Scheme (EBM). The majority of services are not invoiced directly by means of a monetary value but by a system of weighted points. Euro-based charges can be accounted for selected services, such as transportation, documentation, and some screening. To assess the monetary payment in the outpatient setting, the weighted points are usually multiplied by a uniform orientation value, which is defined by the National Association of Statutory Health Insurance Physicians [21]. Weighted points for the year 2010 were multiplied by a uniform orientation value of 0.035048 euros [22].

The utilization of healthcare resources was assessed in terms of the numbers of outpatient visits, number of inpatient visits, number of days in the hospital, number of prescriptions, number of therapeutic devices and remedies, number of days of sick leave payment for the sickness fund, and number of days of rehabilitation. Outpatient visits were approximated by counted dates of invoiced EBM codes. In line with the cost calculation, the asthmarelated resource utilization was calculated as the mean difference in each category between the asthma group and the control group (increment).

Statistical testing was applied by using a $t$ test to determine the significance of differences in healthcare resource utilization and costs.

\section{Results}

A total of 49,668 individuals with ICD-10-GM asthma coding in 2010 are included in the study, as well as 248,340 individuals for the control group approach. The study population consists of $51.5 \%$ females and $48.5 \%$ males; the average age in both groups is 38.5 years.

On average, patients with asthma have more outpatient and inpatient visits, a higher amount of prescriptions for pharmaceuticals and likewise for therapeutic devices and remedies, more days in hospital, more days of sick leave payments, and more days of rehabilitation in 2010 in contrast to the control group (see Table 1). The number of outpatient visits and the number of prescriptions are significant with approximately six more consultations per year and almost twice as many drug prescriptions as compared to their controls.

The calculation of mean annual costs per patient for the asthma group results in total costs of $€ 2,168$ in 2010. Most of these costs are attributable to inpatient care $(29.8 \%)$, outpatient care $(28.9 \%)$, and pharmacotherapy $(25.8 \%)$. Therapeutic devices and remedies $(7.4 \%)$ and sick leave payments $(6.4 \%)$ are also relevant but less important. Rehabilitation costs only account for $1.6 \%$ of the total costs (see Table 2).

In contrast, mean annual costs for an insured person in the control group totaled $€ 1,415$. Inpatient care was also the cost driver $(33.2 \%)$. The share of outpatient care (28.9\%) was equal compared to the asthma group whereas the share of pharmacotherapy $(21.3 \%)$ was slightly lower. The costs for therapeutic devices and remedies $(8.7 \%)$ and for sick leave payments $(6.5 \%)$ also played a minor role. The impact of rehabilitation costs $(1.5 \%)$ on the total costs was negligible.

The control group design enables the calculation of disease-related costs for asthma by subtracting the mean costs per domain of the asthma group from the mean cost per domain of the control group. In contrast to the overall costs per asthma patient, incremental asthma-related costs (see Table 2) are highest for pharmacotherapy with $€ 259$ (34.4\%). Outpatient care $(29.0 \%)$ and inpatient care $(23.4 \%)$ are still major components of the asthma-related total costs. Sick leave payments $(6.2 \%)$ and therapeutic devices and remedies $(5.1 \%)$ have a slightly smaller share than in the overall cost perspective. Rehabilitation remains of minor importance.

Several studies have suggested that asthma-related costs increase with disease severity $[11,13]$. To investigate the impact of disease severity on cost levels, patients were grouped into individuals with intermittent and persistent asthma. According to our classification algorithm, $52 \%$ of the asthma patients have persistent asthma. An additional stratification of patients by gender and age groups provides even more detailed information of the distribution of disease-related costs (see Table 3). Annual total costs and incremental asthma-related costs are higher for patients that were classified as having persistent asthma. For both 
Table 1 Mean healthcare resource utilization in 2010

\begin{tabular}{lcll}
\hline Type of resource utilization & $\begin{array}{l}\text { Asthma group } \\
(n=49,668)\end{array}$ & $\begin{array}{l}\text { Control group } \\
(n=248,340)\end{array}$ & $\begin{array}{l}\text { Incremental asthma-related } \\
\text { healthcare resource utilization } \\
\text { (difference of means) }\end{array}$ \\
\hline Number of all outpatient visits & 16.47 & 10.74 & $5.73^{* * *}$ \\
Number of all hospitalizations & 0.25 & 0.17 & $0.08^{* * *}$ \\
Number of days in hospital & 1.79 & 1.23 & $0.56^{* * *}$ \\
Number of drug prescriptions & 11.22 & 5.79 & $5.43^{* * *}$ \\
Number of therapeutic devices & 1.13 & 0.74 & $0.39^{* * *}$ \\
$\quad$ and remedies & 2.49 & 1.61 & $0.88^{* * *}$ \\
Days of sick leave payment & 0.29 & 0.21 & $0.08^{* * *}$ \\
Days of rehabilitation & & &
\end{tabular}

Significant $\alpha=0.05$

$* * * p$ value $=0.000$

Table 2 Costs of asthma patients and incremental asthma-related costs in 2010 in euros

\begin{tabular}{|c|c|c|c|c|c|c|}
\hline \multirow[t]{2}{*}{ Type of cost } & \multicolumn{4}{|c|}{ Asthma group ${ }^{a}$} & \multirow{2}{*}{$\begin{array}{l}\text { Control group } \\
\text { Mean }\end{array}$} & \multirow{2}{*}{$\begin{array}{l}\text { Incremental asthma } \\
\text { related } \\
\text { Difference of means }\end{array}$} \\
\hline & Minimum & Maximum & Standard deviation & Mean & & \\
\hline Outpatient care & 0 & 34,019 & 904 & 627 & 409 & $218 * * *$ \\
\hline Inpatient care & 0 & 161,855 & 3,044 & 645 & 469 & $176 * * *$ \\
\hline Pharmaceuticals & 0 & 390,423 & 3,213 & 560 & 301 & $259 * * *$ \\
\hline Therapeutic devices and remedies & $-257^{\mathrm{c}}$ & 41,667 & 674 & 161 & 123 & $38 * * *$ \\
\hline Rehabilitation & 0 & 29,321 & 436 & 36 & 21 & $15^{* * *}$ \\
\hline Sick leave payments ${ }^{\mathrm{d}}$ & 0 & 55,132 & 1,647 & 139 & 92 & $47 * * *$ \\
\hline Total & $-3^{\mathrm{c}}$ & 399,180 & 5,740 & 2,168 & 1,415 & $753 * * *$ \\
\hline
\end{tabular}

${ }^{\mathrm{a}} n=49,668,{ }^{\mathrm{b}} n=248,340$, significant $\alpha=0.05 ; * * * p$ value $=0.000$

c Claims data is collected for accounting purposes. Negative costs might occur as a result of reversals and regresses

d A total of 1,087 individuals in the asthma group (2.2\%) and 3,831 individuals in the control group (1.5\%) received sick leave payments

genders, asthma-related costs are highest in the age group over 18 years with persistent asthma.

\section{Discussion}

Considering the widespread prevalence of asthma in the German population, the economic burden of the disease is a significant challenge for the Statutory Health Insurance. Pharmacotherapy and outpatient care are the major cost drivers for incremental asthma-related costs, which is consistent with existing literature [2,13]. The costs for inpatient care are still considerable with $23 \%$ of the asthma-related costs.

Depending on patient characteristics and asthma severity, overall and incremental asthma-related costs might vary substantially. On average, overall total costs per patient with asthma were lowest with $€ 723$ in the group of females from 0 to 5 years with intermittent asthma or wheezing. In contrast, the lowest mean cost for the treatment of asthma is $€ 107$ within the group of females from 6 to 18 years. The highest mean overall incremental asthmarelated costs were found in the group of women with persistent asthma above the age of 18 and amounted to $€ 1,208$

Although randomly selected, the proportion of male and female patients in the study sample reflects the data on 12-month asthma prevalence presented in the literature. In the childhood population, the 12-month asthma prevalence of male patients is higher (3.4-11.8\%) compared to female patients (2.5-9.2\%) [1-3], whereas in the adult population, women are more likely to have asthma with a 12-month prevalence of $6.2 \%$ compared to adult men with $4.2 \%$ [7].

The strength of the present study is its potential to describe the resource utilization and costs of asthma under real-life conditions and for the whole spectrum of services reimbursed by the Statutory Health Insurance system. Moreover, we are able to show that asthma is more expensive compared to the age- and gender-matched sickness fund population. Due to the nature of German sickness fund claims data, the presented resource utilizations and costs provide a complete picture of all health services reimbursed by the sickness fund on the patient level. This is one of the key advantages of this data source for the execution of health economic analysis from the perspective 


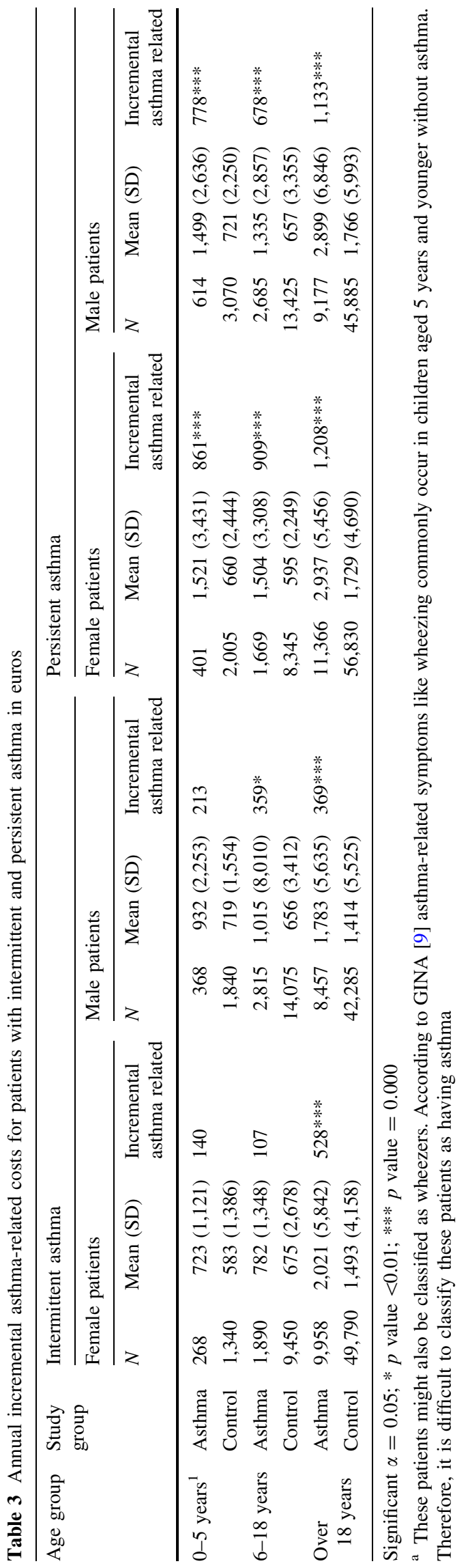

of the sickness fund compared to other primary and secondary data sources [19].

This is the first study for Germany that consistently reports the cost of illness for asthma from the perspective of the SHI using claims data. Stock et al. [2] calculated direct costs of $€ 48.2$ million for asthma-related hospitalization, $€ 62.5$ million for inpatient rehabilitation, and $€ 579.7$ million for asthma-specific medication using claims data and data from national statistics for the year 1999.

Schramm et al. [13] reported direct costs for patients with asthma and seasonal allergic rhinitis in a range from $€ 569$ per adult patient with only seasonal allergic rhinitis to $€ 2,048$ for patients with severe asthma and seasonal allergic rhinitis for the year 2000. Data for cost calculation was collected by patient questionnaires and patients' records. The study population consisted of 500 individuals.

Weinmann et al. [14] investigated the costs of asthma in children resulting in average treatment costs of $\$ 627$ per year. Data was collected from chart review of the involved physicians. Costs were calculated on a 1996 basis and converted to US\$. A total of 76 children with asthma participated in the study.

Weißflog et al. [10] estimated total asthma costs of $€ 2.97$ billion for the German setting in 1996 using data from the AOK sickness fund statistics and the statistical yearbook. Direct asthma costs were $€ 1.92$ billion.

Schulenburg et al. [11] analyzed data from 216 asthma patients in Germany collected by questionnaires in participating doctor's offices. Direct asthma costs of adult patients ranged from $€ 1,060$ to $€ 4,073$ with increasing disease severity. The direct asthma costs for children increased from $€ 1,327$ to $€ 2,460$, respectively. The calculation was based on 1994/95 cost information.

Nowak et al. [5] estimated the costs of asthma for Germany. Direct asthma costs amounted to $€ 1.613$ billion in 1992. Data was collected from available literature sources and extrapolated to the German population.

A recent review from Kirsch et al. [16] estimated annual asthma-specific costs of $€ 445$ to $€ 2,543$ per patient from the social perspective. Cost data was collected from published cost-of-illness studies and adjusted for the year 2010. Direct asthma costs ranged from $€ 175$ to $€ 1,718$ and were reported in detail for outpatient care (€109-€292), inpatient care $(€ 12-€ 100)$, pharmaceuticals $(€ 139-€ 484)$, and rehabilitation (€9-€64). Sick-leave payments (€64$€ 379)$ were also reported, but were not part of the calculation of total asthma costs. The reported cost data provides an estimate of the costs of asthma from a social and the payer's perspective using the available data.

Nevertheless, none of the included studies considered all relevant cost domains and the calculations were mostly based on assumptions or were estimated when specific data were missing or were not detailed enough. The present 
cost-of-illness study fills the gap by considering all relevant cost domains from the perspective of the sickness fund. Because sickness fund claims data contain all reimbursed services attached to single individuals, the results in this study are more precise than the former assumption-based calculations. Moreover, our study provides deeper insights into the distribution of asthma costs and related resource utilizations for different age groups, gender, and disease severity.

The study design with a control group approach is a valid instrument to calculate asthma costs based on the difference in total healthcare costs (incremental costs). These incremental asthma-related costs not only cover direct treatment costs of asthma but also the costs of an increase of co-morbidities and a worsening of already existing co-morbidities. The large asthma group and the even bigger control group with five controls per asthma patient ensure a robust calculation of incremental asthma-related costs [19].

Besides the strengths of the present study, there are some limitations that should be presented. There is only limited socio-economic information available in the claims data of German sickness funds. Information regarding age and gender of the insurant is available, but no further information concerning social status or income. Moreover, information about quality of life and behavior patterns like smoking are not covered in the data [23]. Therefore, a matching of investigated individuals with their controls is only possible by age and gender. Other patient characteristics that might influence resource utilization and reimbursement could not be considered by this approach.

Another limitation associated with claims data analysis in general and with the present study in particular is the absence of clinical information (e.g., results of laboratory test or blood pressure, in this case lung function, allergic status) [24]. Therefore, it is not possible to definitively distinguish between different asthma control stages or severities. As a proxy, we used the claims for asthmarelated medication to approximate the disease severity for each patient.

Inpatient stays in Germany are reimbursed via a diagnosis-related group (DRG)-based system where a fixed amount is paid depending on the DRG per case. The amount differs with some parameters (e.g., the presence of complication), but not with the medication administered during the stay. All medication is reimbursed by the fixed amount per DRG. Therefore, no separate documentation exists within the claims data of a German sickness fund for the administered medication within an inpatient stay [25]. In fact, the applied algorithm to classify asthma severity relies on information of the prescribed medication. Patients with no record of an asthma-specific medication but with an asthma-related inpatient stay were classified as having persistent asthma if the asthma diagnosis was recorded as the primary diagnosis and as having intermittent asthma if the asthma diagnosis was recorded as the secondary diagnosis. There might be cases where this approach leads to a misclassification of the very patient. However, there were only 192 cases where that rule was applied. From a clinical standpoint, these individuals should be classified as patients with persistent asthma.

In practice, health care services in the outpatient setting are reimbursed by a fixed amount paid by the sickness funds to the Associations of Statutory Health Insurance Physicians (KV) on a quarterly basis. This practice limits the costs for outpatient services from the perspective of the statutory health insurance. Nevertheless, Braun et al. [26] recommended a monetary attribution of weighted points of the Uniform Valuation Scheme (EBM) - as adopted in this study - to take opportunity costs into account. Opportunity costs might occur because of more time-consuming services for specific diseases as physicians have only limited timely resources to treat all their patients. However, monetary values for weighted points differ in the accounting practices in the particular administrative districts of the National Associations of Statutory Health Insurance Physicians. Therefore, an average value was used to assess the invoiced Uniform Value Scale points and transform them into a monetary value. Differences from that mean value might occur in some regions and therefore under- or overestimate the monetary value of an invoiced service for a specific region.

Some services in the outpatient setting are only reimbursed once per quarter. Therefore, the calculation of outpatient visits by counting the dates of invoiced EBM codes per patient might result in an underestimation of actual number of outpatient visits. However, these uncounted outpatient visits result in no additional costs from the payer perspective.

Finally, by using only data of the biggest German sickness fund, although nationwide, the results might not be fully representative for the whole German Statutory Health Insurance population.

\section{Conclusions}

Considering the widespread prevalence of the disease in the German population, the economic burden is significant. Incremental asthma-related costs vary substantially according to disease severity and patient characteristics. They increase considerably with higher age and the manifestation of persistent asthma. As for the resource use, the disease results in a significantly higher utilization of healthcare resources, not only drugs but also outpatient care as well as inpatient care. To direct future 
interventions, like disease management programs, further research regarding the diversity of asthma characteristics and especially the identification of high-cost patient groups is needed.

Conflict of interest The study was funded by an unrestricted grant from GlaxoSmithKline.

\section{References}

1. Schlaud, M., Atzpodien, K., Thierfelder, W.: Allergic diseases. Results from the German Health Interview and Examination Survey for Children and Adolescents (KiGGS). Bundesgesundheitsblatt. Gesundheitsforschung. Gesundheitsschutz. 50(5-6), 701-710 (2007)

2. Stock, S., Redaelli, M., Luengen, M., Wendland, G., Civello, D., Lauterbach, K.W.: Asthma: prevalence and cost of illness. Eur. Respir. J. 25(1), 47-53 (2005)

3. Hasford, J., Uricher, J., Tauscher, M., Bramlage, P., Virchow, J.C.: Persistence with asthma treatment is low in Germany especially for controller medication-a population based study of 483,051 patients. Allergy. 65(3), 347-354 (2010)

4. von, M.E., Martinez, F.D., Fritzsch, C., Nicolai, T., Roell, G., Thiemann, H.H.: Prevalence of asthma and atopy in two areas of West and East Germany. Am.J.Respir.Crit Care Med. 149(2 Pt 1), 358-364 (1994)

5. Nowak, D., Volmer, T., Wettengel, R.: Asthma bronchiale-eine Krankheitskostenanalyse. Pneumologie. 50, 364-371 (1996)

6. Heinrich, J., Hoelscher, B., Frye, C., Meyer, I., Wjst, M., Wichmann, H.E.: Trends in prevalence of atopic diseases and allergic sensitization in children in Eastern Germany. Eur. Respir. J. 19(6), 1040-1046 (2002)

7. Koch-Institut, Robert (ed.): Daten und Fakten: Ergebnisse der Studie "Gesundheit in Deutschland aktuell 2010" Beiträge zur Gesundheitsberichterstattung des Bundes. RKI, Berlin (2012)

8. Buhl, R., Berdel, D., Criée, C.-P., Gillissen, A., Kardos, P., Kroegel, C., Leupold, W., Lindemann, H., Magnussen, H., Nowak, D., Kascha-Pfeiffer, D., Rabe, K., Rolke, M., SchultzeWerninghaus, G., Sitter, H., Ukena, D., Vogelmeier, C., Welte, T., Wettengel, R., Worth, H.: Leitlinie zur Diagnostik und Therapie von Patienten mit Asthma. Pneumologie. 60, 139-183 (2006)

9. Global Initiative for Asthma: Global strategy for asthma management and prevention 2014. www.ginasthma.org (2014)

10. Weissflog, D., Matthys, H., Virchow Jr, J.C.: Epidemiology and costs of bronchial asthma and chronic bronchitis in Germany. Dtsch. Med. Wochenschr. 126(28-29), 803-808 (2001)

11. von der Schulenburg, J.-M., Greiner, W., Molitor, S., Kielhorn, A.: Medizinische Ökonomie, Kosten der Asthmatherapie nach Schweregrad, Eine empirische Untersuchung. Med. Klin. 91(10), 670-676 (1996)

12. Statistische Bundesamt: Krankheitskosten in Mio. $€$ für Deutschland. www.gbe-bund.de

13. Schramm, B., Ehlken, B., Smala, A., Quednau, K., Berger, K., Nowak, D.: Cost of illness of atopic asthma and seasonal allergic rhinitis in Germany: 1-yr retrospective study. Eur. Respir. J. 21(1), 116-122 (2003)

14. Weinmann, S., Kamtsiuris, P., Henke, K.D., Wickman, M., Jenner, A., Wahn, U.: The costs of atopy and asthma in children: assessment of direct costs and their determinants in a birth cohort. Pediatr. Allergy Immunol. 14(1), 18-26 (2003)
15. Märtens, P., Lobermeyer, K.: Krankheitskosten-Studie und Kosten-Nutzen-Analyse der spezifischen Immuntherapie bei Asthma. Allergo J. 10, 341-347 (2001)

16. Kirsch, F., Teuner, C.M., Menn, P., Leidl, R.: Costs of illness for asthma and COPD in adults in Germany. Gesundheitswesen. 75(7), 413-423 (2013)

17. Wettengel, R., Berdel, D., Cegla, U., Fabel, H., Geisler, L., Hofmann, D., Krause, J., Kroidl, R.F., Lanser, K., Leupold, W., et al.: Recommendations of the German Respiratory Tract League on asthma management of adults and children. The German Respiratory Tract League. Med. Klin. (Munich). 89(2), 57-67 (1994)

18. Aumann, I., Prenzler, A., Welte, T., Gillissen, A.: Epidemiology and costs of asthma in Germany-a systematic literature review. Pneumologie. 68(8), 557-567 (2014). doi:10.1055/s-00341377225

19. Zeidler, J., Braun, S., "Sekundärdatenanalysen”, Gesundheitsökonomische Evaluationen.In: Schöffski,O., Graf von der Schulenburg, J. M.(eds.), pp. 243-274 Springer, Berlin (2012)

20. Greiner, W., Schöffski, O., "Grundprinzipien einer Wirtschaftlichkeitsuntersuchung," Gesundheitsökonomische Evaluationen. In: Schöffski, O., Schulenburg, J.M. (eds.) pp. 167-191. Springer, Berlin (2007)

21. Prenzler, A., Zeidler, J., Braun, S., von der Schulenburg, J.M.: Bewertung von Ressourcen im Gesundheitswesen aus der Perspektive der deutschen Sozialversicherung. Pharmacoeconomics. Ger. Res. Artic 8(1), 47-66 (2010)

22. Kassenärztliche Bundesvereinigung (Hrsg.)]National Associationof Statutory Health Insurance Physicians (publisher)], "Beschluss des erweiterten Bewertungsausschusses gemäß \$87 Abs. 4 SGB V in seiner Sitzung am 23. Oktober 2008 zur Anpassung des Einheitlichen Bewertungsmaßstabs (EBM) sowie zur Neuordnung der vertragsärztlichen Vergütung im Jahr 2009 [Decision of the Extended Valuation Committee Pursuant To Section 87 Paragraph 4 SGB V [Social Security Code V] in its meeting on 23rd October 2008 on the Adjustment of the Uniform Value Scale as well as the Reorganisation of Remunerating SHI Physicians in 2009]," Deutsches Ärzteblatt, Vol. 105, No. 48, 2008, pp. A 2602-2604

23. Schubert, I., Köster, I., Küpper-Nybelen, J., Ihle, P.: Versorgungsforschung mit GKV-Routinedaten. Bundesgesundheitsbl. 51(10), 1095-1105 (2008)

24. Icks, A., Chernyak, N., Bestehorn, K., Brüggenjürgen, B., Bruns, J., Damm, O., Dintsios, C.M., Dreinhöfer, K., Gandjour, A., Gerber, A., Greiner, W., Hermanek, P., Hessel, F., Heymann, R., Huppertz, E., Jacke, C., Kächele, H., Kilian, R., Klingenberger, D., Kolominsky-Rabas, P., Krämer, H., Krauth, C., Lüngen, M., Neumann, T., Porzsolt, F., Prenzler, A., Pueschner, F., Riedel, R., Rüther, A., Salize, H.J., Scharnetzky, E., Schwerd, W., Selbmann, H.K., Siebert, H., Stengel, D., Stock, S., Völler, H., Wasem, J., Schrappe, M.: Methoden der gesundheitsökonomischen Evaluation in der Versorgungsforschung. Gesundheitswesen 72(12), 917-933 (2010)

25. Bowles, D., Damm, O., Greiner, W.: Gesundheitsbezogene Versorgungsforschung mit GKV-Routinedaten-Grenzen am Beispiel der Prophylaxe venöser Thromboembolien in der Hüft- und Kniegelenkendoprothetik. Gesundh ökon Qual manag. 16(02), 96-107 (2011)

26. Braun, S., Prenzler, A., Mittendorf, T., von der Schulenburg, J.M.: Appraisal of resource use in the German health-care system from the perspective of the statutory health insurance. Gesundheitswesen. 71(1), 19-23 (2009) 